\title{
Histomorphometric Evaluation of Bone-Guided
}

\section{Regeneration in Maxillary Sinus Floor Augmentation Using Nano-Hydroxyapatite/Beta-Tricalcium Phosphate Composite Biomaterial: A Case Report}

\author{
Saulo Henrique Salviano' \\ João Carlos Amorim Lopes ${ }^{2}$ \\ Igor da Silva Brum (10 ${ }^{3}$ \\ Lúcio Frigo (1D ${ }^{4}$ \\ Mario josé dos Santos 5 \\ Sílvio Roberto Consonni (iD) ${ }^{6}$ \\ Jorge José de Carvalho ${ }^{7}$ \\ 'Implantology Department, University \\ São Leopoldo Mandic, Campinas, São \\ Paulo, Brazil; ${ }^{2}$ Implantology Department, \\ Portuguese Catholic University, Lisboa, \\ Portugal; ${ }^{3}$ Implantology Department, \\ State University of Rio de Janeiro, Rio de \\ Janeiro, 20550-900, Brazil; \\ ${ }^{4}$ Periodontology Department, \\ Universidade Guarulhos, Guarulhos, São \\ Paulo, 07023-070, Brazil; ${ }^{5}$ Biology \\ Department, State University of Rio de \\ Janeiro, Rio de Janeiro, 20550-900, Brazil; \\ ${ }^{6}$ Department of Biochemistry and Tissue \\ Biology, Institute of Biology, State \\ University of Campinas (UNICAMP), \\ Campinas, São Paulo, I3083-862, Brazil; \\ ${ }^{7}$ Laboratory of Ultrastructure and Tissue \\ Biology, Department of Histology and \\ Embryology, State University of Rio de \\ Janeiro, Rio de Janeiro, 20550-900, Brazil
}

Correspondence: Igor da Silva Brum Email igor_brum@hotmail.com
Background: The development of techniques in biomaterials design and production added to advanced surgical procedures which enabled better and more predictable clinical outcomes. Maxillary sinus floor augmentation (MSFA) is among the more studied bone-guided regeneration procedure in the literature. The MSFA could be considered the gold standard procedure for bone-guided regeneration as it provides suitable functional and aesthetic solutions to alveolar ridge atrophy due to tooth loss.

Purpose: This study aimed to conduct a detailed histomorphometric evaluation of collagen production in SFAs bone-guided regeneration, using nano-hydroxyapatite/ß-tricalcium phosphate (nano-HA/ß-TCP) composite.

Patients and Methods: A 52-year-old female had the left upper second premolar condemned due to periodontal disease, then a tooth implant replacement was planned. Due to maxillary sinus pneumatization, the MSFA had to be done before implant placement. NanoHA/ß-TCP composite $(2 \mathrm{~g})$ was used in the MSFA procedure. After nine months of the healing process, during the Cone Morse implant installation process, bone samples were collected for histologic analysis (sirius red, hematoxylin/eosin, polarized microscopy). Six months after implant installation, a ceramic crown was installed according to the patient's request.

Results: Proper masticatory function and aesthetics were re-established. The histomorphometric evaluation indicated that nano-HA/ $3-$ TCP composite did not show any area devoid of cellular activity in sirius red or hematoxylin/eosin staining and the percentage (\%) of new bone collagen fibers was achieved using polarization technique evaluation.

Conclusion: According to these results, nano-HA/B-TCP composite presented clinical and histomorphometric properties suit to be used as bone-guided regeneration biomaterial in MSFA. Furthermore, nano-HA/ $\beta$-TCP composite provided a favorable nano-environment to bone cells, enhancing bone matrix production.

Keywords: implants, sinus lift, alloplastic, graft, nanohydroxyapatite, sirius red

\section{Introduction}

The necessity for executing the sinus lift maxilla surgery is still very significant, mainly since many patients extract the teeth from the posterior maxilla region. The responsible technician for the surgery doesn't use any technique of bone guided regeneration, therefore the severe pneumatization maxillary sinus occurs. ${ }^{1}$ Currently, countless techniques may be used for returning masticatory function, 
eg, zygomatic, osteoconduction inter alia, although the most used and documented technique is the lateral window technique because it presents a surgical technique less elaborated and largely known. However, the rupture of the Schneider membrane is a common fact that usually harms the procedure running. ${ }^{2}$

Among the materials available on the market for performing the sinus lift maxilla surgery, we can cite the autogenous, xenogenic, autologous, and alloplastic grafts. In this clinic case presented, it was selected a nano biomaterial with synthetic origin (alloplastic) since this biomaterial class has been presenting countless scientific evidence that it's possible to have greater bone and vascular formation in comparison to the other classes., 3

Bone regeneration has been studied for a long time due to its relevance in human health and complex cell events involved in the process have emerged from this prolific research field. The medical research field is usually linked to pioneering research and testing new types of biomaterials that may promote effective bone regeneration. ${ }^{5}$

Maxillary sinus floor augmentation (MSFA), or maxillary sinus lift, is the clinical procedure in dentistry that is more beneficial in bone-guided biomaterials development that emphasizes bone regeneration. ${ }^{6}$

The use of Bone-graft biomaterials dates back to the 1960s with calcium-phosphate and other absorbable materials to replace bone loss. In the following decade, synthetic polymers, like bioglass, were cataloged as the first synthetic biomaterial enabling adhesive properties to living tissue. Bone morphogenetic proteins (BMPs) were described in the same period and consolidated bone bioengineering as a well-defined research field. In the 1980s bioglass was approved by the Federal Drug Administration (FDA) as the first bioactive material. The last 20 years witnessed the exponential development of different types of bone-graft biomaterials. ${ }^{7}$

Among the recently developed bone regenerationinducing biomaterials, synthetic calcium-phosphate provided a friendly environment for stem cells to grow. Calcium-phosphate in hydroxyapatite (HA) form, mainly found on bone matrix, exhibited strong osteoconduction and osteoinduction properties. ${ }^{8,9}$ However, HA had some limitations in clinical applications: a very slow absorption rate in vivo, that could be as long as 20 years; a weak crack resistance, and a low stimulus to bone tissue regeneration. HA limitations induced active research in calciumphosphate compounds that could improve upon biomaterial absorption and enhance bone cells response. Beta-tricalcium phosphate $\left(\beta\right.$-TCP) best fulfilled these attributes. ${ }^{10}$

The lateral window technique (modified Caldwell-Luc approach) is usually selected for MSFA when considerable bone volume gain is needed. In addition to an adequate surgical approach, a careful biomaterial selection should be observed in these cases. Properties like the frame to withstand mechanical forces and induce a prompt bone tissue regeneration are essential to future implant installation, irrespective of dental implant technique choice (immediate or after bone healing). ${ }^{11}$

MSFA procedure success is related to the amount of vital bone formed after graft maturation and the long-term dental implant survival in this region is related to bone graft maturation as well. ${ }^{12}$

This study aimed to evaluate the clinical results with the dental implants throughout graft technique with nanobiomaterials and using these results to supply a more detailed histomorphometric evaluation of collagen fibrils using different types of staining techniques (Hematoxylin/ Eosin and Sirius red) and microscopy (light and polarized) in MSFA using nano-HA/ $\beta$-TCP composite as a bone graft.

\section{Patients and Methods Patient Data}

A 52 old female non-smoker, medically fit (no systemic disease) and had no bruxism, consulted the Oral Surgery and Maxillofacial Department at São Leopoldo Mandic university (DF, Brazil) in January of 2019. The patient's chief complaint was a missing upper premolar (\#24) and molars (\#26, \#27) that were extracted several years ago due to periodontitis and she was looking for implant replacements. Medical data and complementary exams confirmed normal health conditions. Cone-beam computed tomography (CBCT) confirmed \#24, \#26, \#27 absence and condemned \#25 because did not have bone support. In addition, severe bone loss (mean bone height of $2.0 \mathrm{~mm}$ ) and a pneumatized maxillary sinus were evidenced in CBCT as well (Figure 1). The patient provided informed consent to publish their case details and any accompanying images and institutional approval was not required to publish the case details.

\section{Treatment Planning/Execution}

Treatment (maxillary sinus lift) would start with MSFA using a bone-graft biomaterial in the first surgery stage, followed by two implant installations and \#25 would be 

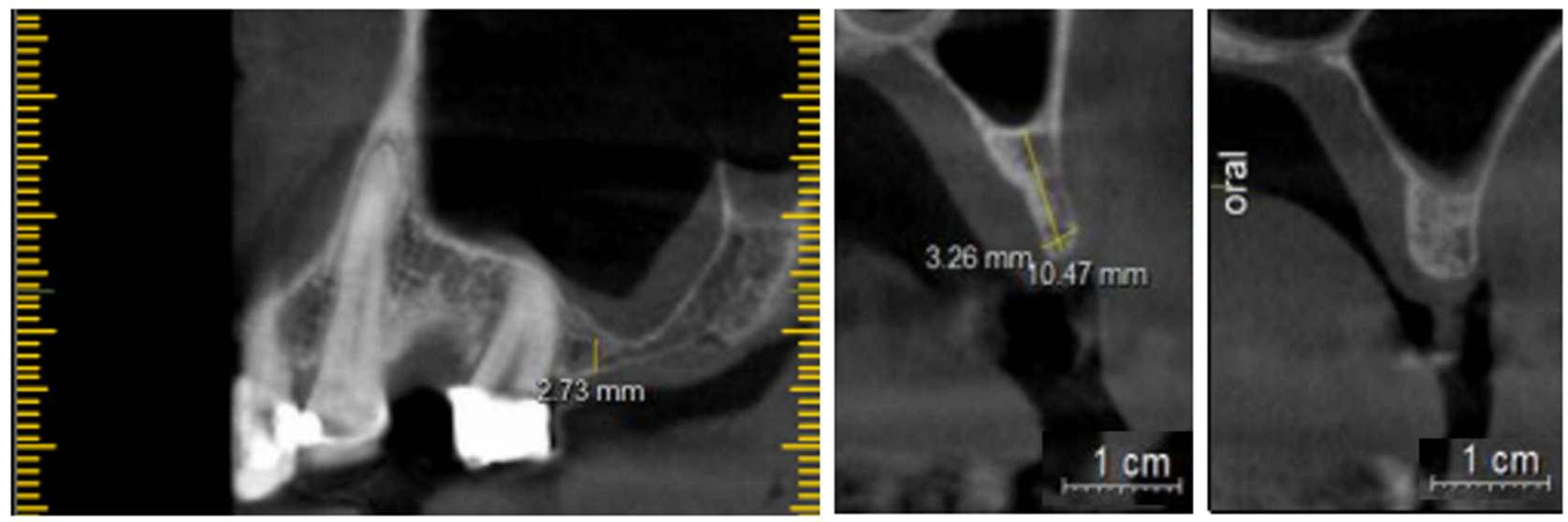

Figure I Cone-beam computed tomography parasagittal section showing pneumatized maxillary sinus and severe crestal ridge bone loss. Dental elements areas 24 and 26.

extracted in the second surgery stage before a final ceramic crown installation. After the patient's accordance and OABR department bio-ethical guideline consent form signing, treatment started.

\section{Surgical Procedures}

All surgical procedures, including follow-up evaluations, were conducted in Oral Surgery and Maxillofacial Department at Brasilia Air Force clinical facility OABR (DF, Brazil).

Amoxicillin (Amoxil ${ }^{\circledR}$, GlaxoSmithKlein, GB) (2g, O. I.) was administered $1 \mathrm{~h}$ previous to surgical procedures and chlorhexidine gluconate $(0.12 \%) \quad$ Periogard $^{\circledR}$, Colgate, USA) mouth rinse was done just before surgery.

Local anesthesia was induced by injection of lidocaine chlorhydrate (2\%) epinephrine 1:100.000 (Alphacaine $100^{\circledR}$, DFL, Brazil). According to Tatum et al (1986), ${ }^{9}$, technique, a horizontal incision, $3 \mathrm{~mm}$ from the mucogingival border, from the tuberal region to the anterior sinus margin was made. Vertical releasing flap incisions were made, exceeding the osteotomy limits, and the mucoperiosteal flap was elevated to access the bone surface. Lateral window osteotomy was performed using \#6 round bur. The sinus membrane (Schneider's membrane) was elevated to biomaterial insertion. Nano-HA/ $\beta$-TCP composite (Blue Bone ${ }^{\circledR}$, Regener, Brazil) was used.

After surgical procedures, the patient was submitted to the radiographic (periapical and panoramic) examination and $\mathrm{CBCT}$ again. Potassium clavulanate/amoxicillin (Clavulin ${ }^{\circledR}, 785 \mathrm{mg}$, Brazil) was prescribed (12/ $12 \mathrm{~h}$ ) for 14 days and precautions were advised: avoid nose blow, cough or sneeze with open mouth simultaneously.
Nine months after MSFA surgery, the patient underwent new local anesthesia and the surgical procedures were conducted to install two Cone Morse implants (Avantt, Systhex ${ }^{\circledR}$, Brazil) in $\# 24$ and $\# 26$ region (3.5 $\mathrm{mm} \times 11.5 \mathrm{~mm}-$ implant dimensions).

Two horizontal incisions in the maxillary ridge crest were made, bone surfaces were exposed and prior to pilot drilling a bone sample for histological analysis was obtained, using a $4 \mathrm{~mm}$ diameter trephine $(\varnothing=4.0 \mathrm{~mm} \times$ length $=6.8 \mathrm{~mm}$ ) (Figure 2).

In constant $0.9 \%$ saline irrigation and suctioning, sequential drilling followed pilot drilling $(800 \mathrm{rpm})$ (BLM 660 model, Driller, Brazil), and implants were installed with a torque of $35 \mathrm{~N} / \mathrm{cm}$. Healing caps were placed, gingival tissue repositioned, and sutured.

Oral antibiotics (Metronidazole 300mg TID and Amoxicillin 500mg TID) for 3 days were prescribed, in

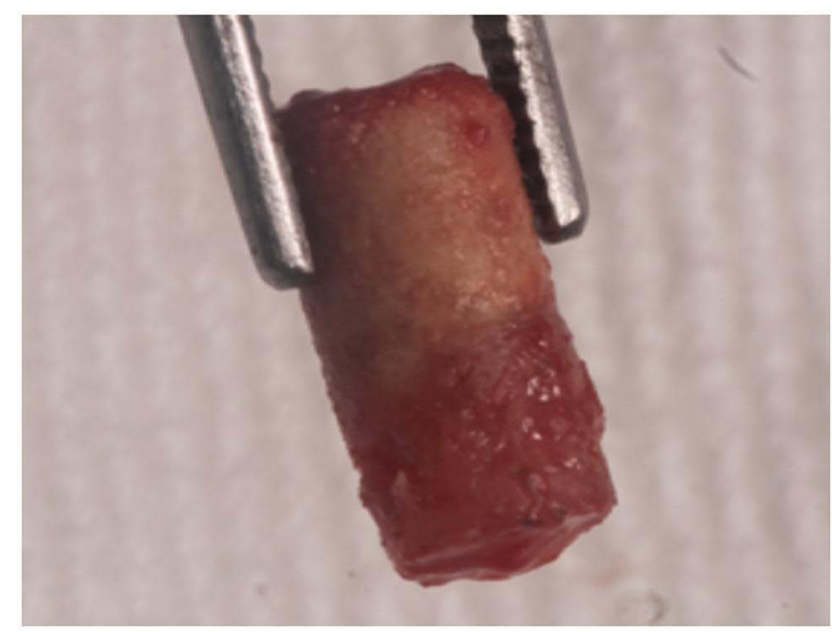

Figure 2 Trephined bone sample assigned to histological procedures and the dental implants inside the graft. 

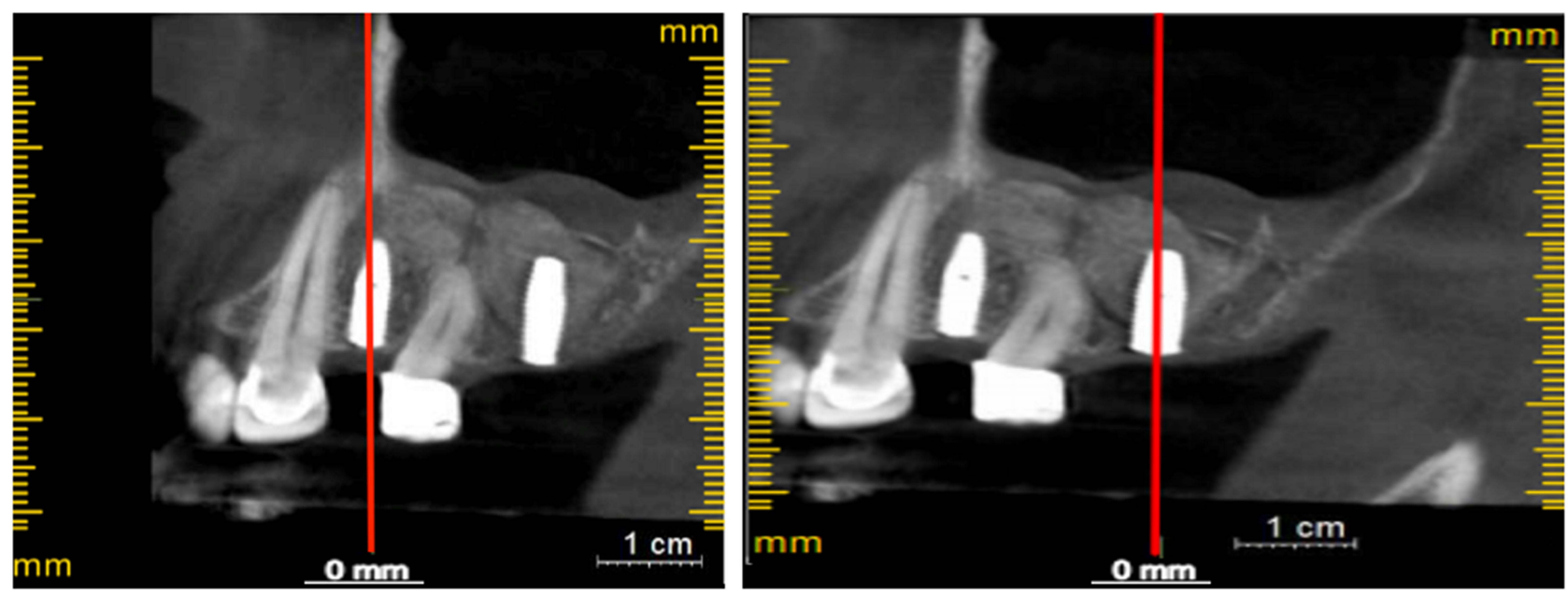

Figure 3 Cone-beam computed tomography parasagittal section showing implants installed after nine months of sinus floor augmentation.

addition to chlorhexidine gluconate $(0.12 \%)$ (Periogard ${ }^{\circledR}$, Colgate, USA) mouthwash for 7 days. A six-month period to osseointegration was awaited (Figure 3).

Six months after implants osseointegration, healing caps were removed, abutments were fitted, an impression was taken and a wax-bite registration was made for an accurate three crown prosthetic confection (\#24, \#25, \#26). After impression, \#25 was extracted and in the following two weeks, three crowns ceramic prosthetic was installed.

\section{Histological Techniques Protocols}

The histological bone sample was soaked in buffered formaldehyde solution (10\%), pH 7.4 for $48 \mathrm{~h}$ prior to demineralization. Phosphate-buffered (0.1M, pH 7.4) EDTA ethylenediaminetetraacetic acid (7\%) (Sigma-Aldrich, USA) for 40 days. The sample was rinsed in tap water, ethanol dehydrated $(75,90,100 \%)$, and clarified in xylene (Sigma-Aldrich, USA).

The sample was rehydrated $(100 \%, 90 \%, 75 \%)$ and embedded in Paraplast ${ }^{\circledR}$ (Sigma-Aldrich, USA) at 650C. Five micrometre sections were obtained, using a microtome (Leica, Germany). Histological sections were collected in slides and divided into two groups assigned to Hematoxylin/Eosin (H/E) and Sirius Red (SR) staining.

For H/E staining, sections received 2 baths of xylene (Sigma-Aldrich, USA), the first one at $60-55^{\circ} \mathrm{C}$ for $5 \mathrm{~min}$ and the second one at room temperature for $20 \mathrm{~min}$. Sections were rehydrated into decreasing concentrations of ethanol $(100 \%$ to $70 \%)$, washed in tap water, bathed for $30 \mathrm{sec}$ in hematoxylin solution (1\%) (Sigma-Aldrich, USA), and washed again. Sections were, then, bathed in eosin $(0.5 \%)$ (Sigma-Aldrich, USA) for $2 \mathrm{~min}$ washed in tap water, immersed in increasing concentrations of alcohol $(100 \%$ to $70 \%)$, and a final alcohol/xylene solution (1:1) bath for $5 \mathrm{~min}$. After drying, slides were coverslipped with Enthelan ${ }^{\circledR}$ (Sigma-Aldrich, USA).

For SR staining, sections received 2 baths of xylene, the first one at $60-55^{\circ} \mathrm{C}$ for $5 \mathrm{~min}$ and the second one at room temperature for $20 \mathrm{~min}$. Sections were rehydrated into decreasing concentrations of ethanol (100\% to $70 \%)$, washed in tap water. Sections were bathed for 15 to 30 min in Sirius Red solution (1\%) (Sigma-Aldrich, MO, USA), washed in running water, dried, and counterstained with a solution of hematoxylin blue (3\%) (Sigma-Aldrich, USA). After drying, slides were coverslipped with Enthellan ${ }^{\circledR}$ (Sigma-Aldrich, USA).

Histological slides were examined and imaged at conventional or polarized light using an upright Nikon E800 microscope (Olympus, Japan) with a P6FL profluorescence camera (Optika, Italy). Five aleatory fields were selected in 100x magnification and analyzed at 200x for collagen clumpiness, density e heterogeneity in each field. Images were analyzed in Image-Pro Plus for Window software, version 7.0.1 (Media Cybernetics) (Figure 4).

\section{Statistical Analysis}

The data were analyzed using one-way ANOVA followed by a Friedman test and Dunn's post-test $(\mathrm{p}<0.05)$. All analyses were conducted with specific software (GraphPad 


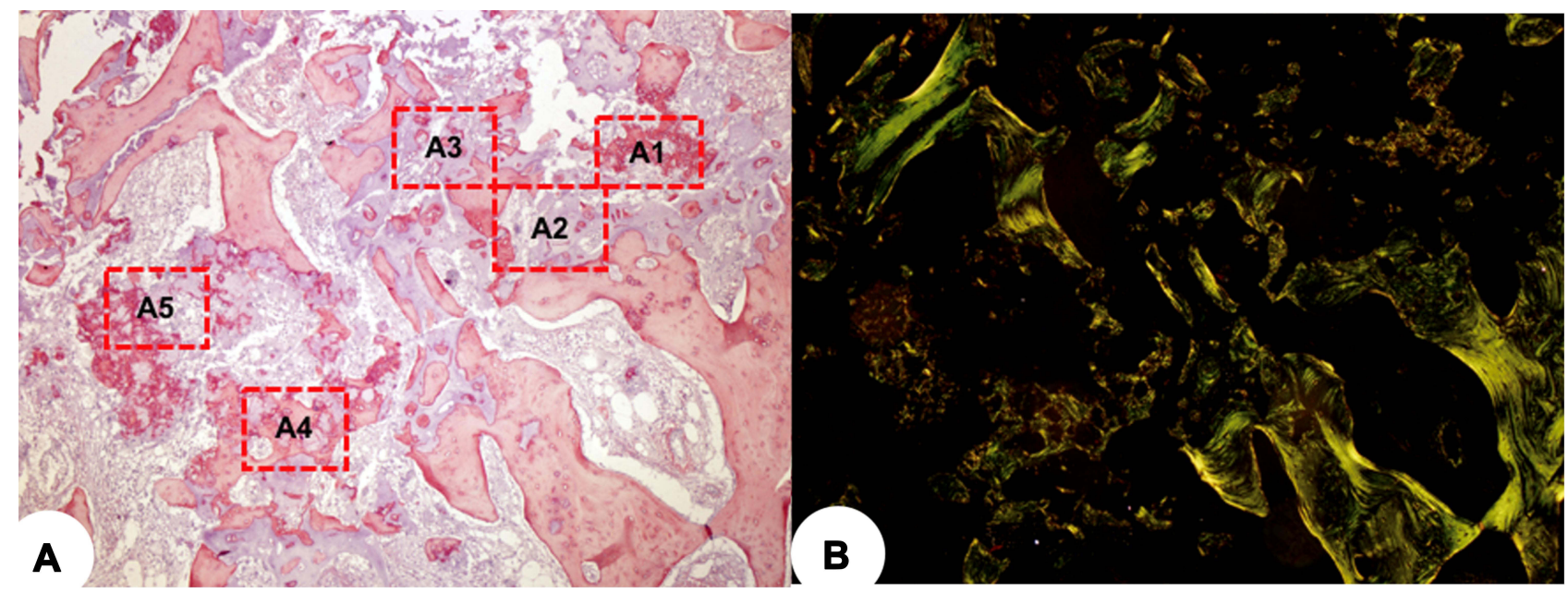

Figure 4 SR staining in bright-field (A) and polarized light microscopy (B) exemplifying five aleatory areas selection (red dotted squares) suit to architecture description and collagen morphometry. Magnification 4x.
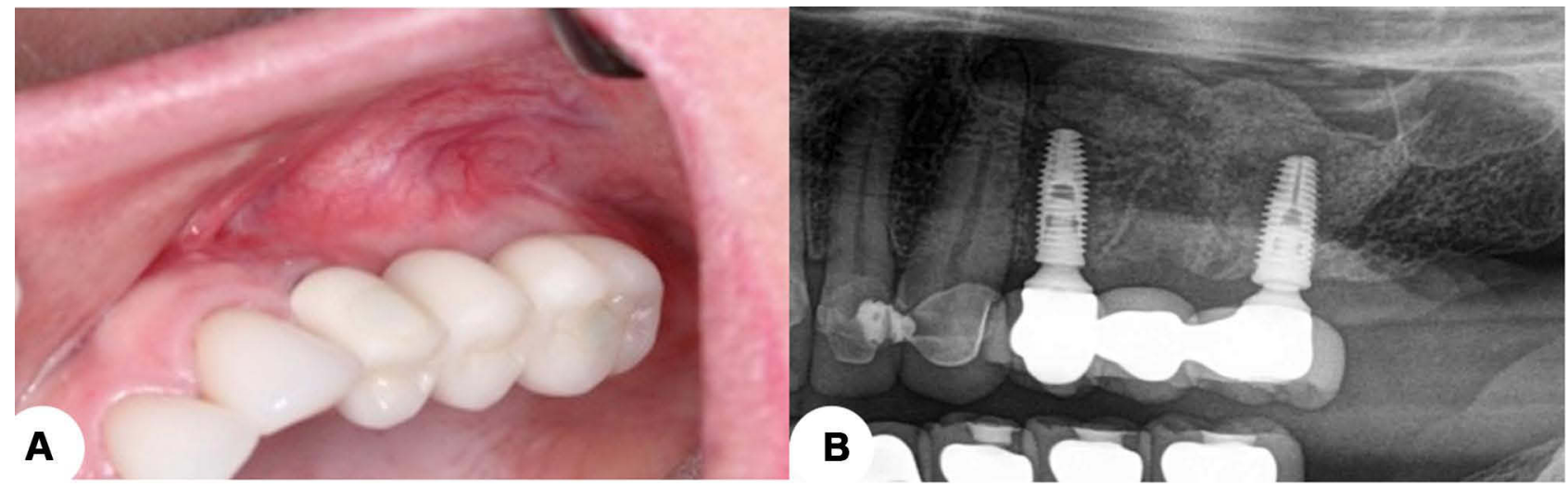

Figure 5 Aesthetics results achieved (A) and radiographic evaluation after three months follow-up (B).

Prism Version 8.0 and BioEstat 5.0). Five areas of the histologic sample were randomly selected, then separately analyzed. The data was generated using (GraphPad Prism Version 8.0 e BioEstat 5.0).

\section{Results}

\section{Outcome and Follow-Up}

After alloy-ceramic crowns were applied, it was fine tuned in the same visit. Implant stability was tested, in addition to occlusion in all mandibular courses, cervical margin adaptation, embrasures, and aesthetics. The final results were highly satisfactory and approved by the patient. The patient was monthly followed-up in the next 6 months, and during this period the implant stability and occlusion were observed. Bone height stability and gingival health were verified as well (Figure 5).

\section{Histological Findings}

HE histological evaluation showed a various amount of newly formed trabecular bones within or adjacent to biomaterial areas, soft tissue, and remaining grafted material particles in the biopsy after 9 months of surgery biopsies. Areas of narrow trabecular bone of varying size with bone lining cells, osteoblasts, and osteocytes, and areas between the biomaterial and soft tissue with osteoclasts were consistently observed in the biopsy. Interestingly the newly formed bone was in direct contact with - or occasionally completely engulfed within biomaterial and only a few osteoclasts were seen close to the bone. The marrow spaces were primarily filled with fibrous connective tissue with no signs of inflammation (Figure 6).

PS staining in conventional and polarized light microscopy evaluation showed both areas of low birefringence, an indicative of immature bone with disorganized collagen 

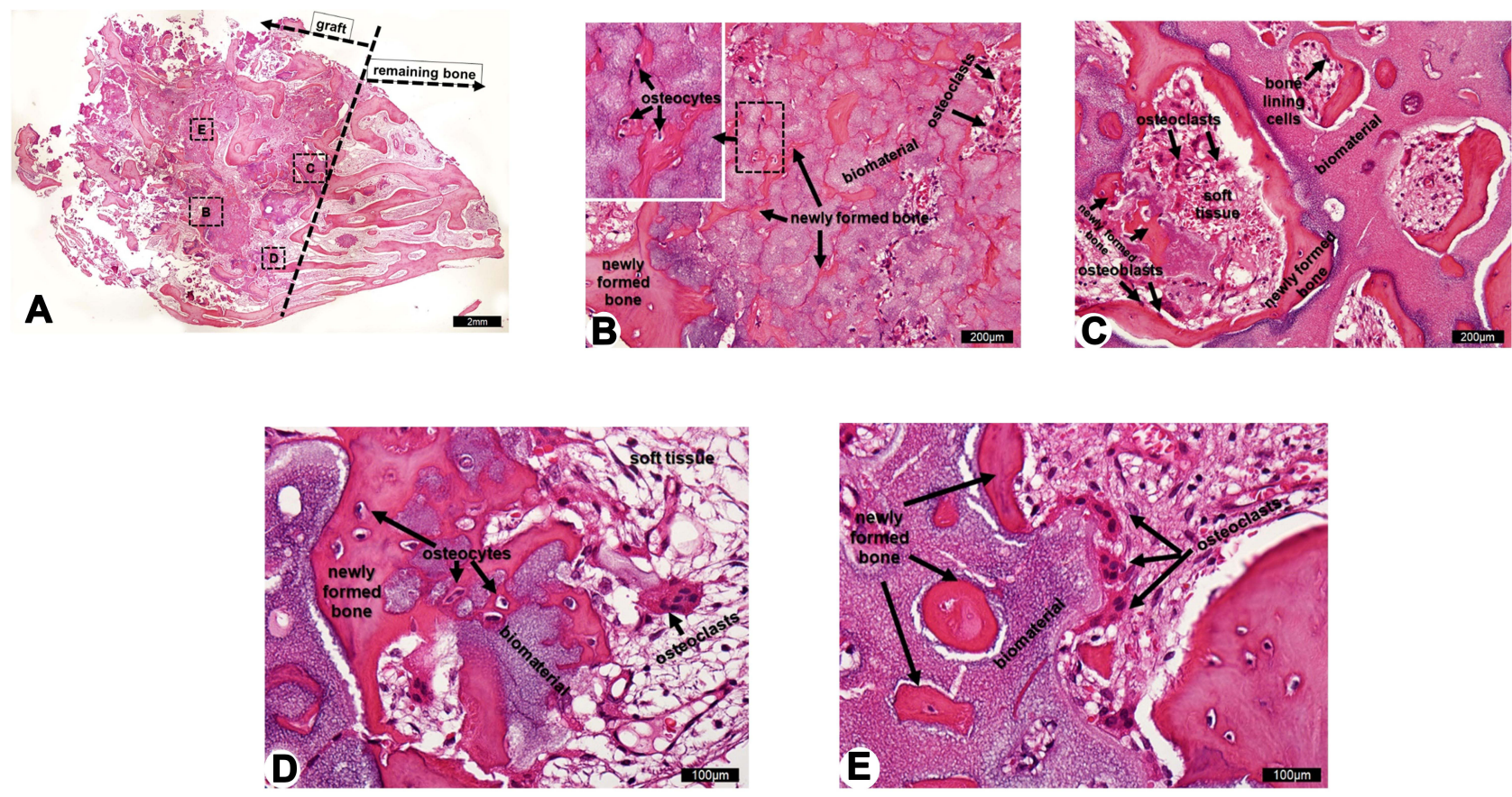

Figure 6 Representative photomicrographs of longitudinal section of biopsy after 9 months of surgery. (A) Observe remaining bone and graft area of the whole biopsy in low magnification. Square dotted lines indicate areas (B-E) photographed in higher magnification. (B) Note a flocculated basophilic biomaterial with osteocytes within narrow newly formed trabecular bone and osteoclasts at periphery. (C-E) Newly formed trabecular bones within or adjacent to biomaterial areas can be observed. Bone lining cells, osteoblasts and osteocytes are observed associated to narrow trabecular bone, while osteoclasts are mostly seen associated between biomaterial and soft tissue. Hematoxylin/Eosin staining. Scale bar $A=2 \mathrm{~mm} ; B$ and $C=200 \mu \mathrm{m} ; \mathrm{D}$ and $E=100 \mu \mathrm{m}$.

bundles, as well as areas of high birefringence in the newly formed bone, and indicative of mature lamellar bone with high aggregation and organization of collagen bundles (Figure 7).

Statistical analysis, using the Friedman test, of SR staining selected areas in polarized microscopy indicated no differences in collagen density, clumpiness, and heterogeneity (Figure 8).

\section{Discussion}

Provide adequate bulk bone support to replace alveolar crest loss or sinus pneumatization is the main goal of a multitude of surgical techniques and biomaterials. Our case report illustrated the selection of a traditional surgical approach and innovative biomaterial to fulfill functional and aesthetics demanded by the patient. ${ }^{13}$

In a recent review of the trends toward biomaterials development, HA-based composites were considered the ideal framework for new bone formation. ${ }^{14}$

Synthetic-HA chemical properties are considered more stable than natural (naive) bone HA, so it is the slowest absorption rate in bone regeneration/remodeling. Aggregating a faster-absorption calcium-phosphate, like $\beta$-TCP, would provide a more uniform timeline bone regeneration/remodeling rate. These characteristics guided the Nano-HA/ $\beta$-TCP composite design used in this case report and already pre-clinically and clinically tested by the authors. ${ }^{8,15}$

In addition to clinical outcomes and follow-up that were considered highly satisfactory, histological/histomorphometric evaluation was conducted using traditional staining techniques indicated to tissue architecture $(\mathrm{H} / \mathrm{E})$ and collagen fibers (PS). Despite PS-polarizing microscopy's shortcomings, if compared to more advanced techniques (immunohistochemistry) or chemical analysis, it is reliable and very easy to use. If PS-polarizing microscopy does not provide detailed collagen chemical characteristics, it does provide thorough access to the spatial architecture of the fiber's arrangement and enables its quantitation. ${ }^{16,17}$

Kubasiewicz et al evaluated four different types of biomaterial osteoconductors potential: micro-HA, nanoHA, nano-HA/ $\beta-T C P$, and nano-HA+collagen membrane. Histomorphometric analysis indicated that every experimental group tested induced bone regeneration, ranging from $34.2 \%$ to $44.4 \%$, against $13 \%$ of the control group (no biomaterial used). ${ }^{19}$ Moreover, in the nano-HA group, no evidence of biomaterial was found, in contrast with all 


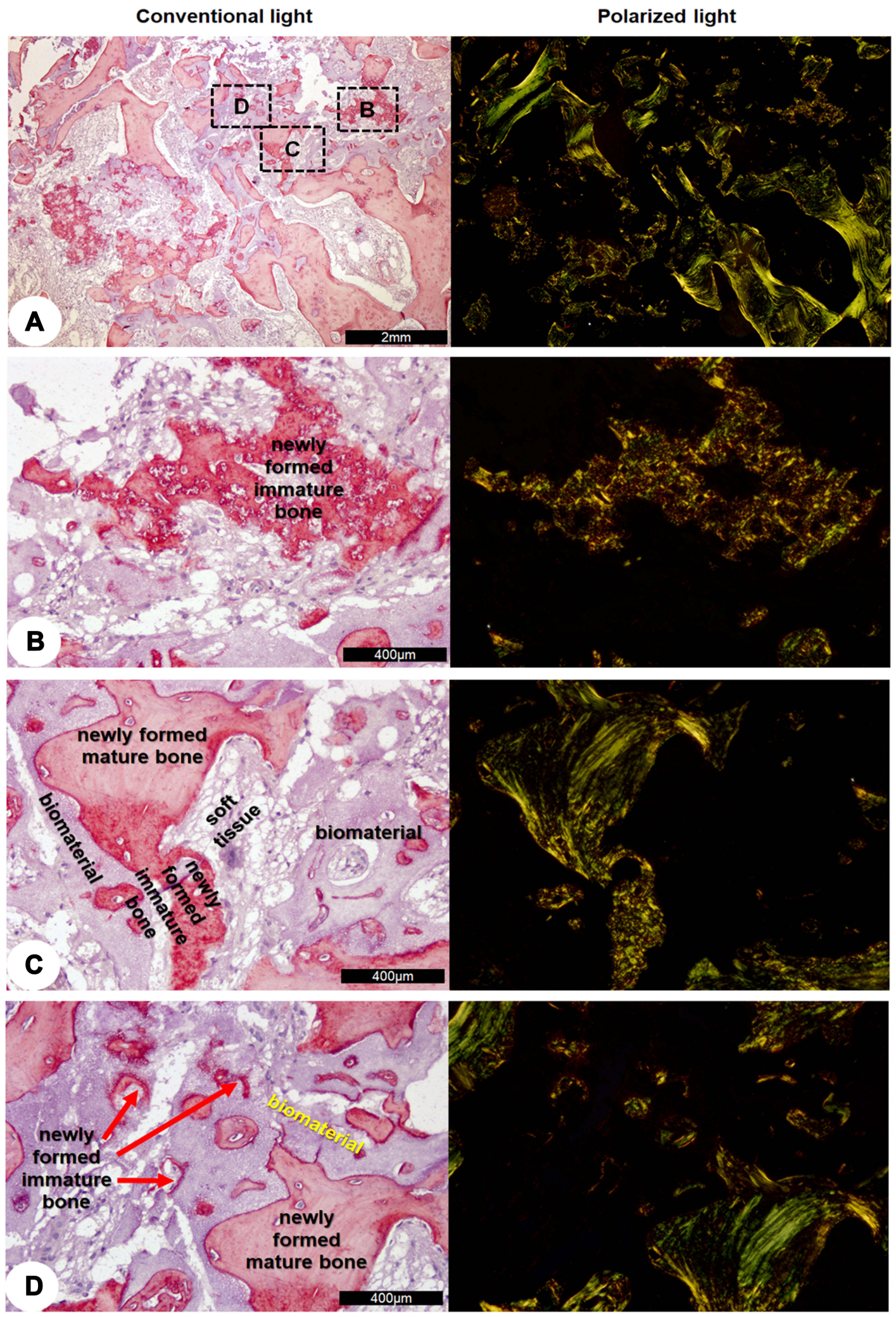

Figure 7 Representative photomicrographs of biopsy after 9 months of surgery at bright-field (left panel) and polarized light microscopy (right panel). (A) Low magnification of biopsy. Square dotted lines indicate areas (B-D) photographed in higher magnification. (B-D) Newly formed immature or mature bones within or adjacent to biomaterial areas close to soft tissue can be observed. The same areas under polarized light show typical collagen bundle (appearing as bright lines) are well arranged, suggesting mature lamellar bone, while collagen fibers undulations suggests immature (non-lamellar) bone. The dark area shows no collagen organization. Sirius Red staining. Scale bar $\mathrm{A}=$ $2 \mathrm{~mm}$; B-D $=400 \mu \mathrm{m}$. 
A

Density (mean)

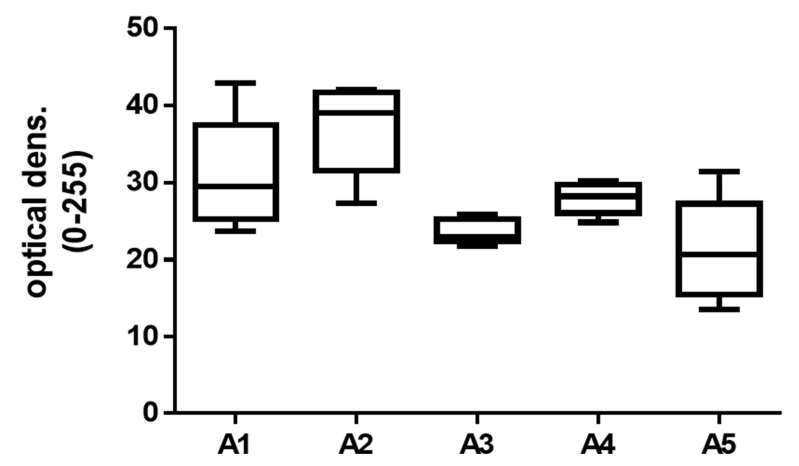

B Clumpiness

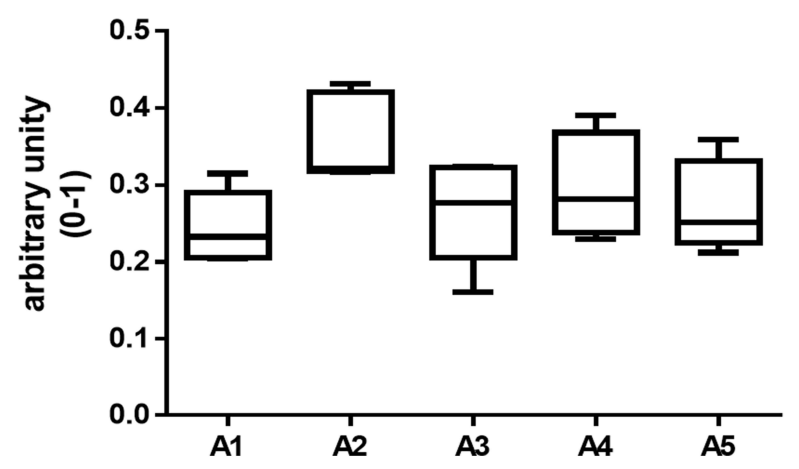

C

Heterogeneity

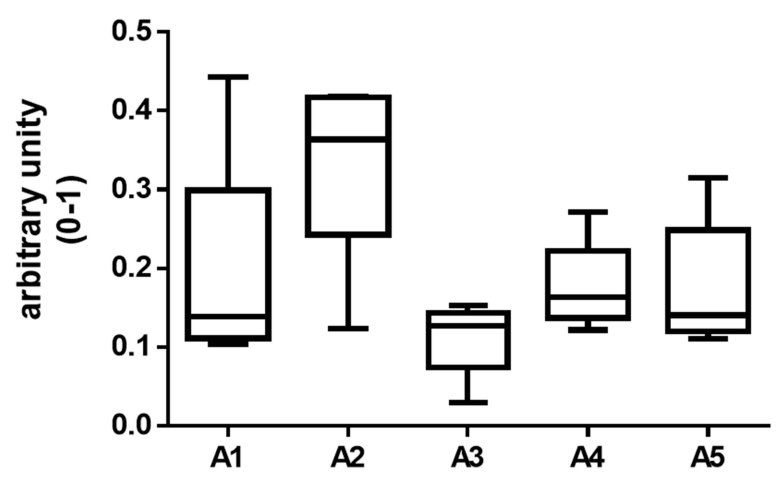

Figure 8 Graphics showing statistical analysis of SR staining in polarized microscopy in five aleatory selected areas (AI-A5) that evaluated mean collagen density (A) $(P=0.0342)$, collagen fibrils clumpiness $(B)(P=0.1257)$ and collagen heterogeneity $(C)(P=0.0976)$.

other test groups in which varying amounts of biomaterial could be found. These results indicate more proper resorption of nano-HA. ${ }^{20} \mathrm{~A}$ similar finding was observed in our histologic evaluation. Nano-HA/ $\beta$-TCP composite could be observed as a highly active inducer of new bone formation. Newly formed immature bone was observed in the borders of and inside particles, suggesting substitution is in due course.

Studies conducted to evaluate MSFA, using different types of biomaterials, concluded that new bone volume affects important clinical outcomes. ${ }^{21,22}$ Also, histological/ histomorphometric studies highlighted different cell responses among HA, $\beta$-TCP, and HA/ $\beta$-TCP composite experimental groups. They concluded that HA/ $\beta$-TCP composite induced more new bone formation than HA or $\beta$-TCP alone. Histological evaluation of the herein case report supports these findings, suggesting highly active areas of bone formation/remodeling as the new bone matrix, osteoclasts, osteocytes, and bone-lining cells were evident. $^{23,24}$
MSFA case report using micro-HA concluded that low-rate biomaterial resorption impaired an effective cell interaction. ${ }^{24}$ Bone defects evaluation in dogs, using micro-HA, carbonate apatite $\left(\mathrm{CO}_{3} \mathrm{Ap}\right)$ and $\beta$ TCP, concluded that $\mathrm{CO}_{3} \mathrm{Ap}$ induced a higher production of new bone, due to its superior osteoconduction and osteoinduction potential. ${ }^{25}$ The similar potential of osteoconduction and osteoinduction was demonstrated in studies using the composition of nano-HA $(80 \%)$ and $\beta$-TCP (20\%). Histomorphometric/histologic evaluation indicated a higher number of bone cells (Osteocytes, osteoblasts, and osteoclasts), in addition to a higher number of blood vessels and a new bone matrix. $^{26-29}$

\section{Conclusion}

Based on case report outcome and follow-up in addition to histologic/histomorphometric evaluation, it was possible to conclude that nano-HA/ $\beta$-TCP composite was a suit biomaterial indicated to maxillary sinus floor augmentation. Nano-HA 
$/ \beta$-TCP composite provided a favorable nano-environment to bone cells, enhancing bone matrix production.

\section{Funding}

This research received financial support from the Brazilian agencies CNPq, FAPERJ, CAPES, LUBT, Fisclinex and UERJ.

\section{Disclosure}

The authors report no conflicts of interest in this work.

\section{References}

1. Juzikis E, Gaubys A, Rusilas H. Uses of maxillary sinus lateral wall bony window in an open window sinus lift procedure: literature review. Stomatologica. 2018;20(1):14-21.

2. Kim JM, Sohn DS, Heo JU, Moon JW, Lee JH, Park IS. Benefit of the replaceable bony window in lateral maxillary sinus augmentation: clinical and histologic study. Implant Dent. 2014;23(3):277-282. doi:10.1097/ID.0000000000000070

3. Glenske K, Donkiewicz P, Köwitsch A, et al. Applications of metals for bone regeneration. Int $J$ Mol Sci. 2018;19(3):826-832. doi:10.3390/ijms19030826

4. Pappalardo S, Puzzo S, Carlino V, Cappello V. Bone substitutes in oral surgery. Minerva Stomatol. 2007;56(10):541-557.

5. Huang S-H, Hsu -T-T, Huang T-H, et al. Fabrication and characterization of polycaprolactone and tricalcium phosphate composites for tissue engineering applications. J Dent Sci. 2017;12(1):33-43. doi:10.1016/j.jds.2016.05.003

6. Raghoebar GM, Onclin P, Boven GC, Vissink A, Meijer HJA. Longterm effectiveness of maxillary sinus floor augmentation: a systematic review and meta-analysis. $J$ Clin Periodontol. 2019;21:307-318. doi:10.1111/jcpe.13055

7. Koons GL, Diba M, Mikos AG. Materials design for bone-tissue engineering. Nat Rev Mater. 2020. doi:10.1038/s41578-020-0204-2

8. da Silva Brum I, de Carvalho JJ, da Silva Pires JL, de Carvalho MAA, Dos Santos LBF, Elias CN. Nanosized hydroxyapatite and $\beta$-tricalcium phosphate composite: physico-chemical, cytotoxicity, morphological properties and in vivo trial. Sci Rep. 2019;9 (1):19602. doi:10.1038/s41598-019-56124-4

9. Pilipchuk SP, Plonka AB, Monje A, et al. Tissue engineering for bone regeneration and osseointegration in the oral cavity. Dent Mater. 2015;31(4):317-338. doi:10.1016/j.dental.2015.01.006

10. Kim HD, Amirthalingam S, Kim SL, Lee SS, Rangasamy J, Hwang NS. Biomimetic materials and fabrication approaches for bone tissue engineering. Adv Healthcare Mater. 2017;6 (23):1700612. doi:10.1002/adhm.201700612

11. Silva LD, de Lima VN, Faverani LP, de Mendonça MR, Okamoto R, Pellizzer EP. Maxillary sinus lift surgery-with or without graft material? A systematic review. Int J Oral Maxillofac Surg. 2016;45 (12):1570-1576.

12. Lopes LF, da Silva VF, Santiago JF Jr, Panzarini SR, Pellizzer EP. Placement of dental implants in the maxillary tuberosity: a systematic review. Int J Oral Maxillofac Surg. 2015;44:229-238. doi:10.1016/j. ijom.2014.08.005

13. Tatum H Jr. Maxillary and sinus implant reconstructions. Dent Clin North Am. 1986;30(2):207-229.

14. Silva LD, de Lima VN, Faverani LP, de Mendonça MR, Okamoto R, Pellizzer EP. Maxillary sinus lift surgery-with or without graft material? A systematic review. Int J Oral Maxillofac Surg. 2016;45 (12):1570-1576.
15. Ramesh N, Moratti SC, Dias GJ. Hydroxyapatite-polymer biocomposites for bone regeneration: a review of current trends. $J$ Biomed Mater Res B Appl Biomater. 2018;106(5):2046-2057. doi:10.1002/ jbm.b.33950

16. da Silva Brum I, Frigo L, Lana Devita R, et al. Histomorphometric, immunohistochemical, ultrastructural characterization of a nano-hydroxyapatite/beta-tricalcium phosphate composite and a bone xenograft in sub-critical size bone defect in rat calvaria. Materials (Basel). 2020;13(20):4598. doi:10.3390/ma13204598

17. Lattouf R, Younes R, Lutomski D, et al. Picrosirius red staining: a useful tool to appraise collagen networks in normal and pathological tissues. $J$ Histochem Cytochem. 2014;62(10):751-758. doi:10.1369/0022155414545787

18. Kubasiewicz-Ross P, Hadzik J, Seeliger J, et al. New nano-hydroxyapatite in bone defect regeneration: a histological study in rats. Ann Anat. 2017;213:83-90. doi:10.1016/j. aanat.2017.05.010

19. Coelho PGB, Souza MV, Conceição LG, Viloria MIV, Bedoya SAO. Evaluation of dermal collagen stained with picrosirius red and examined under polarized light microscopy. An Bras Dermatol. 2018;93 (3):415-418. doi:10.1590/abd1806-4841.20187544

20. Kermani F, Kargozar S, Tayarani-Najaran Z, Yousefi A, Beidokhti SM, Moayed MH. Synthesis of nano HA/ $\beta$ TCP mesoporous particles using a simple modification in granulation method. Mater Sci Eng C Mater Biol Appl. 2019;96:859-871. doi:10.1016/j. msec.2018.11.045

21. Rojbani H, Nyan M, Ohya K, Kasugai S. Evaluation of the osteoconductivity of $\alpha$-tricalcium phosphate, $\beta$-tricalcium phosphate, and hydroxyapatite combined with or without simvastatin in rat calvarial defect. J Biomed Mater Res A. 2011;98(4):488-498. doi:10.1002/ jbm.a.33117

22. Olaechea A, Mendoza-Azpur G, Valle O, et al. Biphasic hydroxyapatite and B-tricalcium phosphate biomaterial behavior in a case series of maxillary sinus augmentation in humans. Clin Oral Implants Res. 2019;30(4):336-343. doi:10.1111/clr.13419

23. Ramírez-Fernández MP, Calvo-Guirado JL, Maté-Sánchez Del Val JE, Delgado-Ruiz RA, Negri B, Barona-Dorado C. Ultrastructural study by backscattered electron imaging and elemental microanalysis of bone-to-biomaterial interface and mineral degradation of porcine xenografts used in maxillary sinus floor elevation. Clin Oral Implants Res. 2013;24(5):523-530. doi:10.1111/j.16000501.2011.02414.x

24. Mendes LD, Bustamante RP, Vidigal BC, et al. Effect of amount of biomaterial used for maxillary sinus lift on volume maintenance of grafts. J Clin Exp Dent. 2020;12(9):e830-e837.

25. Sato N, Handa K, Venkataiah VS, et al. Comparison of the vertical bone defect healing abilities of carbonate apatite, $\beta$-tricalcium phosphate, hydroxyapatite and bovine-derived heterogeneous bone. Dent Mater J. 2020;39(2):309-318. doi:10.4012/dmj.2019-084

26. Lambert F, Bacevic M, Layrolle P, Schüpbach P, Drion P, Rompen E. Impact of biomaterial microtopography on bone regeneration: comparison of three hydroxyapatites. Clin Oral Implants Res. 2017;28 (10):e201-e207. doi:10.1111/clr.12986

27. Shin HI, Sohn DS. A method of sealing perforated sinus membrane and histologic finding of bone substitutes: a case report. Implant Dent. 2005;14(4):328-333. doi:10.1097/01.id.0000188465.93052.cd

28. Berglundh T, Lindhe J. Healing around implants placed in bone defects treated with Bio-Oss. An experimental study in the dog. Clin Oral Implants Res. 1997;8(2):117-124. doi:10.1034/j.16000501.1997.080206.x

29. Albrektsson T, Johansson C. Osteoinduction, osteoconduction and osseointegration. Eur Spine J. 2001;10(Suppl 2):S96-101. doi: $10.1007 / \mathrm{s} 005860100282$ 


\section{Publish your work in this journal}

The International Medical Case Reports Journal is an international, peer-reviewed open-access journal publishing original case reports from all medical specialties. Previously unpublished medical posters are also accepted relating to any area of clinical or preclinical science. Submissions should not normally exceed 2,000 words or 4 published pages including figures, diagrams and references. The manuscript management system is completely online and includes a very quick and fair peer-review system, which is all easy to use. Visit http://www.dovepress.com/testimonials.php to read real quotes from published authors. 